\title{
Developing a Forest Data Portal to Support Multi-Scale \& Multi-Disciplinary Decision Making
}

\begin{tabular}{|c|c|}
\hline Journal: & $\begin{array}{l}\text { Journal of Selected Topics in Applied Earth Observations and Remote } \\
\text { Sensing }\end{array}$ \\
\hline Manuscript ID: & JSTARS-2011-00256 \\
\hline Manuscript type: & $\begin{array}{l}\text { Interoperability Architectures and Arrangements for Multi-Disciplinary } \\
\text { Earth Observation Systems and Applications Special Issue }\end{array}$ \\
\hline $\begin{array}{l}\text { Date Submitted by the } \\
\text { Author: }\end{array}$ & 30-Sep-2011 \\
\hline Complete List of Authors: & $\begin{array}{l}\text { McInerney, Daniel; European Commission, Joint Research Centre } \\
\text { Bastin, Lucy; Joint Research Centre of the European Commission, } \\
\text { Institute for Environment and Sustainability } \\
\text { Diaz, Laura; Universitat Jaume I, Institute of New Imaging } \\
\text { Technologies (INIT) } \\
\text { Barredo, Jose; Joint Research Centre of the European Commission, } \\
\text { Institute for Environment and Sustainability } \\
\text { San-Miguel-Ayanz, Jesus; Joint Research Centre of the European } \\
\text { Commission, Institute for Environment and Sustainability }\end{array}$ \\
\hline Keywords: & Forestry \\
\hline
\end{tabular}




\title{
Developing a Forest Data Portal to Support Multi-Scale and Multi-Disciplinary Decision Making
}

\author{
Daniel McInerney, Lucy Bastin, Laura Díaz, Jose I. Barredo, \\ and Jesús-San-Miguel Ayanz
}

\begin{abstract}
Forests play a pivotal role in timber production, maintenance and development of biodiversity and in carbon sequestration \& storage in the context of the Kyoto Protocol. Policy makers and forest experts therefore require reliable information on forest extent, type and change for management, planning and modeling purposes. It is becoming increasingly clear that such forest information is frequently inconsistent and unharmonised between countries and continents. This research paper presents a forest information portal that has been developed in line with the GEOSS and INSPIRE frameworks. The web portal provides access to forest resources data at a variety of spatial scales, from global through to regional and local, as well as providing analytical capabilities for monitoring and validating forest change. The system also allows for the utilisation of forest data and processing services in other thematic areas. The web portal has been developed using standardised Web services to facilitate accessibility, interoperability and data transfer.
\end{abstract}

\section{Index Terms}

Forest monitoring, interoperability, Earth observation, map viewer, metadata.

D. McInerney (daniel.mc-inerney@jrc.ec.europa.eu), Jose I. Barredo and Jesús San-Miguel-Ayanz are with the Joint Research Centre of the European Commission, Institute for Environment and Sustainability (IES), LMNH - FOREST (TP 261), Via E. Fermi, 274921027 Ispra (VA), Italy, Phone: +39 0332783047.

Lucy Bastin is on secondment from Aston University at the Joint Research Centre of the European Commission, Institute for Environment and Sustainability (IES), GEM (TP44), Via E. Fermi, 274921027 Ispra (VA), Italy.

L.Díaz is with the Intitute of New Imaging Technologies, Universitat Jaume I, Castellon.

Manuscript received September 30 2011; revised ... 


\section{INTRODUCTION}

The Global Earth Observation System of Systems (GEOSS) is being organised by the Group of Earth Observation (GEO), which is an umbrella group that was established in 2005 and includes over 40 international organisations, 62 nations and the European Union. The GEOSS strategy seeks to organise existing systems and services to promote interoperability using existing standards [1]. GEOSS itself is structured into nine societal benefit areas (SBAs) [2], with forestry included specifically within the Agricultural benefit area. However, [3] noted that forest information and observations are required by all nine SBAs, e.g. for the definition of landcover/vegetation type within the Agriculture, Ecosystems and Biodiversity SBAs, for the calculation of biomass/carbon storage assessments in the Climate and Energy areas, as a factor in hydrological modeling for the Water area, and as a source of data on landcover change arising from clearfellings or natural hazards. The forest sector is therefore cross-thematic, with changes in forest structure and composition influencing all SBAs and creating a need for readily accessible and usable forest data at a range of scales from local through to national and global.

Forest data and statistics are typically compiled by Forest Authorities through national forest inventory (NFI) programmes, which collect in-situ information including estimates of forest area, species composition and growing stock [4]. These data are used for strategic planning and production forecasting at national and regional levels, but they are also used to generate indicators for compliance with international reporting requirements, such as the United Nations Food and Agriculture Organization (FAO) Forest Resource Assessment, the Ministerial Conference on the Protection of Forests in Europe and the United Nations Framework Convention on Climate Change Land-use, Land-use Change and Forestry [5], [6].Within the context of the post-Kyoto climate process, national governments are required to maintain a forest carbon accounting system that considers changes in forest stock at the sub-hectare level.

Closely related to this are the UN Reducing Emissions from Deforestation and Forest Degradation (REDD) Programme and the GEO Initiative on Forest Carbon Tracking (GEO FCT). The latter supports countries that wish to implement a national Monitoring, Reporting and Verification (MRV) system though the combined use of satellite, airborne and in-situ data [7]; [8]. These systems would ultimately form the components of a global network of MRV systems, which should be fully interoperable within a distributed network of geographic information 
infrastructures (GIIs).

Additionally, the Global Observation of Forest and Land Cover Dynamics (GOFC-GOLD) is another international effort that seeks to provide timely information on forest and vegetation cover from space-based and in-situ data in order to facilitate better understanding of the terrestrial carbon budget. It was originally set up by the Committee on Earth Observation Satellites (CEOS), but is now an integral part of the Global Terrestrial Observing System (GTOS) [9] [10].

Despite these initiatives, it is acknowledged that there is frequently a distinct lack of harmonised forest information available at regional or continental scales and that in many cases data are scattered, incomplete or not readily comparable [11]. On the one hand, this can be caused by differences in forest definitions between countries [12], but from a technological point of view it is often due to the lack of inter-connection between the systems and data structures established at local, regional and global levels. Initiatives such as the INSPIRE Directive [13], GEOSS and the EuroGEOSS project are making advances to standardise access to spatial information as well as providing common infrastructures allowing data to be discovered, shared and re-used.

More broadly, the increasing use of geo-spatial Web-based standards defined by the Open GeoSpatial Consortium (OGC) has enabled systems to become far more interoperable in general, and has greatly assisted in the exchange of spatial data. These standards apply specifically to the publication of metadata within catalogues, but are also widely used to disseminate spatial data through standard-based web services. The most important services related to this paper include Web Map Services (WMS), Web Feature Services (WFS) and Web Coverage Services (WCS). The WMS standard provides access to maps of spatially referenced data that are rendered using a pictorial format, (e.g., PNG, GIF or JPEG) through simple browser requests submitted using Uniform Resource Locators (URLs) [14]. WMS are widely employed for easily-accessible cartographic visualisations of data stored on a server. WFS and WCS allow a client to retrieve and update not just images, but geospatial data itself. WFS is used for vector spatial data and acts primarily as a feature access service, while the WCS specification allows the exchange of gridded or coverage data that may also have a temporal dimension. In other words, in contrast to the simple image provided by the WMS, the user receives a collection of coordinate pairs or numeric pixel values which can be used in analytical tasks. Thus WFS and WCS can be used as downloading data services, and are frequently consumed by Web Processing Services (WPS) and within Web-based modeling activities. 
From this growing culture of standardised, interoperable Web Services and data formats has arisen the concept of the Model Web an approach to the encoding and wrapping of processing algorithms which allows scientific models to be exposed as Web Services in a flexible, distributed architecture [15]. Using orchestration tools, such as The Invisible Modelling Environment (TIME) and Taverna, these models can then be combined into complex workflows and executed over a distributed architecture. A workflow may itself be exposed as a single Web Service where the user only interacts with the initial inputs and the final outputs.

The practical challenges of achieving genuine interoperability of data, concepts and information models in an open system setting, and across multiple disciplines, are significant. However, the delivery of the Model Web and its components has the potential to extend and enhance scientific modelling by easing the integration and flexible re-use of models and code for decision making, while increasing the reproducibility and transparency of research by providing clear and repeatable provenance information for models and their outputs.

There is an increasing need to develop a network of such interoperable spatial information components that provide access to forest data in a standardized way. This is required for multiscale MRV, but it is equally necessary for Web-based analysis and spatial modeling. This paper describes the components of a system that has been developed within the context of the EuroGEOSS project (http://www.eurogeoss.eu) to deliver forest resources, such as data on forest type and fire events, at a range of spatial scales. This data can be used by forest experts to answer questions relating to the extent and rate of forest change at various spatial scales, as well as the impacts of forest fires on protected areas and tree species. At regional and national levels in Europe, the system is underpinned by the European Forest Data Centre (EFDAC), European Forest Fire Information System (EFFIS) and national data centres. At a global scale, forest data are derived from the Forest Resource Assessment (FRA) exercise on which FAO coordinates its efforts with the TREES-3 project based at the European Commission Joint Research Centre (EC-JRC) [16]; [17].

The objective of this paper is to present new components which access and utilise distributed information on forest resources at different spatial scales and to demonstrate how they can be integrated into geographic information infrastructures. This has been achieved through the development of new forest information infrastructures and Web-based analytical tools that can be used to monitor and quantify forest change at a range of spatial scales. This objective is 
illustrated through the development of an INSPIRE compliant metadata catalogue for discovery of forest information, development of a Web map viewer based on OGC-standard Services and deployment of Web-based forest analysis and modeling tools.

\section{USE CASE SCENARIOS}

\section{A. Study Areas}

Within the context of the EuroGEOSS project, the research presented in this paper focuses on European forestry and environmental data, with specific use-case scenarios developed for Spain. The global dimension of this research is emphasized by the scalability of the forestry data portal and is also demonstrated by its easy incorporation into the Web-based land cover validation tool which is designed to cover the tropical regions of Africa, Latin America and South-East Asia.

\section{B. Core Data Services}

The use-case scenarios presented within this paper are dependent on a number of core European forest data centres, which include the European Forest Data Centre (EFDAC), the European Forest Fire Information System (EFFIS) and the e-Forest platform. These services are managed on an operational basis by the EC-JRC (http://forest.jrc.ec.europa.eu) and provide up-to-date and reliable data on the state of European forest resources which are required for the development of environmental polices at a pan-European level. At a global scale, the TREES-3 action of the Joint Research Centre supplies data services which focus specifically on the mapping and monitoring of landcover change over time, using medium-resolution satellite imagery.

The EFFIS was established in 2002 by the EC-JRC as part of the EU Forest Focus regulation (EC-2152/2003). It is a modular Web-based information system that provides up-to-date fire danger forecast information to national authorities and EC services. It complements existing information collated by national forest authorities and civil protection services to provide harmonized pre- and post-fire information at a pan-European level [18]. In the pre-fire phase, the EFFIS provides access to pan-European harmonized maps of fire danger indices. These are calculated using meteorological forecast data and are updated on a daily basis and published within the information system [19]. In the post-fire phase, the system provides access to a range of historical fire information. 
The EFDAC was established in 2005 following an agreement to establish a number of European Data Centres between the Group of Four (Go4), namely the European Commission Directorate-General Environment (ENV), EC-JRC, EuroSTAT and the European Environment Agency (EEA). It has been established to supply European Union decision-makers with processed, quality checked and timely policy-relevant forest data and information within the EU. In the longer-term, the EFDAC will provide more harmonized and integrated information at panEuropean, regional and local scales. The forest geo-spatial information maintained within the EFDAC is derived from the classification of satellite imagery, the analysis of satellite derived forest maps, and field based assessments of forest resources from European National Forest Inventories (NFI) as well as forest information from various research projects, national forestry correspondents and international forest data sources.

The e-Forest platform is a recent addition to the EFDAC, and consists of a harmonised spatial database of detailed forest resource information derived from National Forest Inventories (NFI) EU Member States. The NFIs are based on sampling frames that collect qualitative and quantitative field measurements, which include species occurrences, detailed measurements of trees (tree diameters and heights). The platform provides a harmonized database of forest information, which can be used to produce forest parameter estimates from pan-European level through to national and regional levels.

The three abovementioned systems publish a range of forest related spatial data that include forest cover maps, tree species distributions, information on forest health and forest fire data (e.g. location and extent of forest fires). All of these data are available as standard OpenGeoSpatial Web Services (OWS), including Web Map Service (WMS); Web Feature Service (WFS) and Web Coverage Service (WCS). These services are all used by the portal discussed within this paper for viewing and analytical purposes.

The TREES-3 Action1 provides quantitative measurements and mapping of changes in forest resources to support maintenance and development of EU policies related to global environmental and forestry issues. The focus of TREES-3 is on Eurasian boreal forests and tropical forests, including the Caribbean and Pacific regions. Thus, while the overall scope of the project is global, there are important thematic and geographic overlaps with the data services mentioned above. TREES-3 also addresses forest cover and cover change issues related to EU commitments to Multilateral Environmental Agreements, especially to UN conventions such as the UNFCCC, 
UNCCD, UNCBD, and the UN Forest Forum, as well as Action Plans such as on Forest Law Enforcement Governance and Trade (FLEGT).

In order to document forest cover change, the TREES-3 project gathers landcover information for $20 \mathrm{~km}$-square sample sites at over 4000 lat-long degree confluence points in the tropical zones of Africa, Asia, and South America [17]. This exercise constitutes the tropical section of the Global Forest Resources Assessment programme1 which is coordinated by the Food and Agricultural Organization of the UN [?]. Multi-spectral imagery for each confluence point [20] is processed and classified using object-based techniques to generate homogeneous landcover segments whose geometry is consistent between the sample years (e.g. 1990 / 2000) [21]; [22]. The classified segments have historically then been validated by forestry experts from the relevant countries using an editing tool written in IDL [23] thus combining specialist knowledge at the EC-JRC and expert knowledge from the countries concerned. The designated national experts verify the content of each landcover segment using high-quality imagery (currently derived from Landsat) as contextual information. It is this validation process for which the Web-based tools described later have been developed, in order to build on the success of the stand-alone IDL application while widening access to the editing tools.

The landcover data derived from the TREES-3 action permits the tracking of areas of rapid forest change and produces statistically valid estimates of cover change from the mid 1970s to 2005-10. TREES-3 products are used as inputs for future climate change impact scenarios and provide a basis for developing input into the Kyoto Protocol process. Biomass maps and carbon emission/storage estimates are produced for selected forest ecosystems.

In addition to the services described above, a number of Spanish data service providers publishing data at national and regional levels are integrated within the analysis relating to biodiversity and protected areas. The main data provider is the Spanish Observatory of Natural and Protected Areas (Observatorio de los Espacios Naturales Protegidos del Estado Español), more commonly referred to as EuroParc.

\section{Approach: Architecture design adding multidisciplinary capabilities}

This section describes the software architecture within which we deploy and integrate the three Web-based applications of the EuroGEOSS Forest Data Portal. These include the Metadata Catalogue, Web-based Viewer and the TREES-3 Web-based validation tool. A schematic overview of 
the system is provided in Figure 1 that demonstrates how the components can be used to search, view and analyse forest data as well as providing the capabilities to combine these resources with those from other thematic areas, such as Droughts, Agriculture or Biodiversity.

The Forest Data Portal was developed on a CentOS Linux environment platform with the individual components developed using Free OpenSource geospatial libraries and programs. The Apache HTTP server (http://httpd.apache.org/) was used with the Apache Tomcat Servlet engine and were used to run the individual Web-based components. The Metadata Catalogue was developed using GeoNetwork version 2.6.3 (http://geonetwork-opensource.org). This version was selected as it is more readily compliant with INSPIRE compared to previous versions and it also includes support for metadata internationalization with access to Google translation Web services. Geoserver (http://www.geoserver.org) was set up for the specific purposes of publishing specific EuroGEOSS forest data layers.

The Web-based viewer was developed using JavaScript libraries. The OpenLayers (http://www.openlayers.org) JavaScript library was used to develop the underlying mapping framework, while ExtJS (http://www.sencha.com) and GeoEXT (http://www.geoext.org) were used to create a sophisticated Web-based mapping interface. The TREES-3 validation tools employ the same suite of JavaScript libraries, with the addition of PostgreSQL and PostGIS in the back-end for the management of the vector spatial database.

The forest Web Processing Services (WPS) were implemented using PyWPS [24], [25], a Python implementation of the WPS Standard. It allows for the development of geo-processing tools with the seamless use of the Geospatial Data Abstraction Library (GDAL) (http://www.gdal.org), GRASS-GIS [26], R-Statistics [27] as well as other Python packages, such as NumPy. In addition, the system offers a Publication Service called the GEOSS Service Factory (GSF) [28]. This service aims at maintaining geospatial information infrastructures assisting users to publish content in existing data services. The GSF hides the complexity of standard interfaces and offers a unique entry point for content to be delivered. GSF is implemented as a WPS and it is included in the user workflow to publish generated information, for instance WPS outputs as standard data services (WMS, WFS). 


\section{RESUlts}

1) Increasing Content Visibility: The EuroGEOSS Forest Metadata Catalogue was developed as a central information component containing metadata concerning spatial and non-spatial forest information. It provides a means of increasing the visibility and retrieval of forest data. The catalogue was populated with contents from EFDAC, EFFIS and eForest. Global forestry information was also included, based on data from the FAO FRA 2010 Statistics, Eurostat and MCPFE. The metadata were imported using DC and ISO XML files and also by harvesting external Metadata Catalogues using the CSW ISO protocol, OAI DC protocol and the OGC OWS services via GetCapabilities requests which can be performed asynchronously or in realtime. This procedure was successfully tested for three catalogues that included the EC-JRC EFDAC Metadata Catalogue and the IDEE Data and Services Catalogues.

As part of the requirements, the catalogue was developed to include a comprehensive list of metadata subthemes for publishing and searching metadata. These subthemes include: global forestry; forest biodiversity; forest condition; forest fires; forest information systems; forest inventory; forest mapping; forest products and forests \& climate change. Additional customisations were made to the Metadata catalogue to provide direct links from the metadata page to the map viewer, with the option of automatically loading specific layers into the map viewer, using either a specific WMS layer end point or a WMS url. It is also possible to access the catalogue, either for harvesting purposes using CSW (catalogue services for the Web) or directly through a Web interface. In addition, the catalogue is registered as a federated catalogue of the EuroGEOSS Discovery Broker, which increases its visibility to scientists from other research disciplines.

2) Map Viewers: The Web-based map components of the EuroGEOSS project are described in two parts: the client Web-based Map Viewer with the combined Web Processing Services and the TREES-3 validation tool. The novel developments of each component are described in turn and a description of how they are used within scenarios is also provided.

The Web-based Map Viewer provides the capabilities to view, query and overlay forestry, biodiversity and drought related information that are served as WMS and WFS layers.

The specific functionality that the viewer provides includes the addition or removal of layers; retrieval of feature information; connecting to distributed WMS servers, viewing metadata 
(summary \& full) and controlling the WPS analytical tools, which is described subsequently. The novel features of the viewer is the possibility for users to readily connect to a wide range of WMS servers, which range in thematic and geographic scope, from forestry to biodiversity at European, national and regional scales. The viewer also gives users the option of to digitizing features on the map canvas, and allowing these features to be subsequently used within the WPS. Figure 2 presents the Forest Map Viewer displaying the WPS outputs.

3) Web-2.0 and OpenSearch Integration: The Web has become a collaborative environment, where increasing numbers of Web-based social networks and active users act as a data providers, representing another massive repository of geo-referenced data of great potential value.. This information, while highly heterogeneous in its nature, quality and formats, provides a complementary view to scientific data and can provide better insights into the social impact of environmental and other phenomena [29]. To exploit this rich resource, we have developed another discovery service that provides a homogeneous search interface to retrieve content from social networks and crowd sourcing platforms, called the Web 2.0 Broker [29]. The Web 2.0 Broker implements the OpenSearch Geo-Time standard interface specification. To access this new discovery service we have designed and developed a web client module to be included in the Forestry Portal. This client, developed with Google Web Toolkit technology, offers a userfriendly interface to facilitate users access to the functionality of the Web2.0 Broker in a simple and visual way.

\section{A. Web-based GeoProcessing}

Two Web Processing Services (WPS) were integrated into the Map Viewer to provide specialized geo-analytical tools for forestry experts. They were specifically developed to allow scientists to address specific questions on forest change using a standardized approach for analyzing and interpreting the data which permits the results to be comparable and reproducible. The specific questions that the services were used to answer were:

- To monitor the impact of forest fires in protected areas;

- To assess the extent of forest change between two time periods. 
1) Use Case 1: Forest Fires in Protected Areas: In recent years, forest fires have affected approximately 300,000 ha of forest every year across Mediterranean countries [30]. This continues to pose a significant threat to the vegetation structure and biodiversity within protected areas. Consequently, there is a need for scientists and resource managers to be able to effectively assess the impact of forest fires on protected areas and this can be achieved through the use of standardized processing and reporting tools. The resulting information can be used by scientists and managers to determine the spatial distribution of forest fires within protected areas and to assess the spatial composition of the burnt areas (forest species and/or landcover). Such information would allow them to better implement post-fire management plans and to allocate available resources.

Figure 3 presents the workflow using the Business Processing Modelling Notation (BPMN) and provides an overview of the geo-processing workflow implemented within the processing services. The principal inputs are the EFFIS Burnt Areas and the Spanish designated protected areas (e.g. Natura2000 sites, national parks etc), which are both published as WFS layers. The WPS can be run at different scales, from local through to regional and or national and the process is carried out using vector spatial analysis.

Within this WPS, the user is required to select the Area Of Interest or Protected Area by means of an interactive selection within the map canvas or from the list of attributes from the Protected Area. Subsequently, the user launches the WPS, which carries out the vector intersection and overlay of the two input features. The resulting intersected geometries are rendered to the map canvas, with associated summary statistics and attributes presented to the user as combination of tables and maps. On completion of the process, the user is given a further choice to launch the GSF to publish the WPS data outputs to the EuroGEOSS Forest GeoServer and the associated Forest Metadata Catalogue.

2) Use Case 1: Monitoring Forest Change: The monitoring of forest change is becoming increasingly important at a range of scales from local through to national and global. There is an increasing requirement by Government and Forest Authorities to quantify the changes in forest cover and forest carbon stocks, and these metrics are frequently aggregated to estimate the countries anthropogenic forest-related greenhouse emissions. Due to the differences in forest definitions and forest inventory methods used by countries, it is quite challenging to obtain harmonized results that can be directly compared between countries. This can be overcome by 
the use of forest cover maps that are derived from satellite image maps that have been produced using consistent methodologies at European scale.

The EC-JRC has produced wall-to-wall forest maps covering Europe for the reference years of 2000 and 2006 [31]; [32] using a semi-automatic classification approach to map forest areas from medium resolution satellite imagery (Landsat TM/ETM+; SPOT 4/5 \& IRS-LISS 1C). The resulting map products are wall-to-wall forest cover maps covering Europe. Currently, in order to utilise these data for analysis, users are required to download, tile and process these data with specialized GIS software. However, the service described within this section, provides scientists and citizens with a standardized and easy approach to retrieve, process and interpret the data through a Web-browser.

The second WPS analyses the extent of gain, loss and stability of forest areas within a specified AOI. In this use-case, the forest data are WCS layers of the EC-JRC Forest Map produced for the years 1990, 2000 and 2006 at a resolution of 25-m.

Within this WPS, the user is required to digitize an AOI and then to select the year of analysis (e.g. Time-0 and Time-1). The WPS then processes the Forest Map WCS, in order to compute the percentage change in forest area between the two periods. The results are displayed to the user as graphs and statistical summaries, as well as an output WMS map showing the areas of forest loss, gain and stability between the two time periods. This computation of the changes in forest area provides forest managers and policy makers with important information relating to areas that are subject to deforestation. A detailed description of the workflow is presented in the BPMN diagram (Figure 4).

As in the other use-case scenario, the user is given the possibility to invoke the GSF to publish the WPS outputs as new content available in the system data services. The GSF publishes the result for further visualization in the existing WMS implemented with Geoserver and for discovery purposes in the CSW implemented with Geonetwork.

3) Web-based validation tool: A Web-based editing tooll has been developed to support the TREES-3 expert validation process described in Section 2.2, again using OGC standards for data exchange formats and geospatial Web services. The tool allows a registered expert to view and query the status of their allocated sites (completed or in progress), and to select segments where particular types of change have occurred, for overlay on ancillary contextual data. The editing and validation process is carried out entirely online, using a PostGIS database which is exposed 
via a Geoserver Web Feature Service and Web Map Service.

National experts log in to view the location and status of their assigned sample sites within a zoomable, draggable map. Ancillary and contextual information can be added from publicly available global mapping sources. When a site is selected for editing, data from two different years are displayed at once (Figure 5). The landcover segments are shown on the right, and the available satellite imagery is shown on the left. Segment labels are edited onscreen by selecting the required areas, selecting the new landcover from a predefined legend, and submitting the change via an HTTP request to a PostGIS database on the server. When the national expert has completed their changes, the site can be signed off as completed from this page.

The user may select specific landcover segments interactively onscreen, or can submit a query for segments which fulfil specific conditions (for example, segments can be selected where a specific type of landcover change has occurred). The geometries of any selected segments are retrieved as GML or GeoJSON from the underlying Web Feature Service, meaning that they can be overlaid on selected contextual data or, in a separate tab, on Google Earth imagery. In the example below, the EuroGEOSS Map Viewer (section 3.2) is integrated as an extra tab, allowing various WMS layers to be selected as the backdrop for these segment outlines.

\section{Discussion: Future Scope}

Within this paper we have highlighted the importance of being able to access forest resource information so that it can be used by scientists and decision makers in an interoperable manner to answer multi-disciplinary questions. We have described a new Forest Data Portal that fulfills this role as a means of searching for data (and metadata), viewing it in conjunction with data from different disciplines and providing functionality for users to analyse these data using Web services.

The Forest data portal has been developed using Open Standards, which is an advantage to bridge the gaps between differing spatial scales, semantic concepts and disciplines. Furthermore, this allows for powerful and flexible analysis of forest related data to answer multi-disciplinary research questions. Following international directives like INSPIRE, we have provided the system with services that allow interoperable access to discover, view, download as well as geoprocessing functionality. Furthermore with the extension of this architecture with a publication service we also assist users to participate more actively in the maintenance of the infrastructure, increasing 
the visibility and availability of content by simplifying the process of publishing content.

The integration of Volunteered Geographic Information (VGI) from Web2.0 technologies offers a unique way of harnessing a vast amount of contextual information from sources such as Flickr, Twitter or YouTube. These sources are rapidly evolving and it is clear that they can be used to complement scientific data and Web processing tool chains.

The TREES-3 validation tool provides a global context of this research and is a central link between the EuroGEOSS Forest Data portal and the UN FAO databases that are being developed within the context of the EuroGEOSS project. The suite of tools described here illustrate a robust framework by which comprehensive forest information can be collected, analysed and easily accessed in an interoperable and consistent way, at a variety of spatial scales ranging from the local expert right up to the global decision maker. It also lends itself to being integrated within GII from other thematic areas for more integrated, multi-disciplinary research tasks.

The Web Processing Services that are presented within this research provide users with robust tools that yield consistent and accurate results of forest change. The advantage of deploying them as WPS is that they can be used within more complex workflows. For instance, the analysis of the forest change database could be considered as a pre-processing step with the outputs used as inputs into other WPS models.

Society and the scientific community increasingly need to be able to access distributed information on the environment, be it forestry, agriculture, droughts or biodiversity. While the access to these data is of fundamental importance, their value is increased when they are used within GII for geo-processing or modeling to answer scientific questions and support decision making. This process could be implemented at any scale from local through to global.

\section{ACKNOWLEDGMENT}

This work has been financially supported by the European Commission FP7 Project nr. 226487 called EuroGEOSS.

\section{REFERENCES}

[1] S. Nativi, P. Mazzetti, H. Saarenmaa, J. Kerr, and E. O. Tuama, "Biodiversity and climatge change use scenarios framework for the geoss interoperability pilot process," Ecological Informatics, vol. 4, pp. 23-33, 2009.

[2] C. Lautenbacher, "The global earth observation system of systems: Science serving society," Space Policy, vol. 22, pp. 8-11, 2006. 
[3] H. Olsson, M. Nilsson, and M. Persson, "Geoss possibilities and challenges related to nation wide forest monitoring," in Proc. of ISPRS Commission VII Mid Term Symposium, 2002.

[4] E. Tomppo, K. Schadauer, R. McRoberts, T. Gschwanter, K. Gabler, and G. Ståhl, "Introduction," in National Forest Inventories Pathways for Common Reporting, E. Tomppo, T. Gschwanter, M. Lawrence, and R. McRoberts, Eds. Heidelberg: Springer, 2010, ch. 1.

[5] Food and A. O. of the United Nations. (2011) Global forest resources assessment 2010. Internet draft. [Online]. Available: http://www.fao.org/docrep/013/i1757e/i1757e.pdf

[6] M. C. on the Protection of Forests in Europe (MCPFE), "State of europe's forests 2007: the mcpfe report on sustainable forest management in europe," Ministerial Conference on the Protection of Forests in Europe Liaison Unit Warsaw, 2007.

[7] M. Brady, "The geo initiative on forest carbon tracking: Towards the implementation of a global forest carbon tracking system," in Proc. Monitoring Forest Carbon Stocks and Fluxes in the Congo Basin.GOFC-GOLD Report No. 44, vol. 1, Brazzaville, Congo, Feb 2010.

[8] — - "The role of national demonstrators in the geo forest carbon tracking task," in Proc. Monitoring Forest Carbon Stocks and Fluxes in the Congo Basin.GOFC-GOLD Report No. 44, vol. 1, Brazzaville, Congo, Feb 2010.

[9] J. Townsend and C. Justice, "Towards operational monitoring of terrestrial systems by moderate-resolution remote sensing," Remote Sensing or Environment, vol. 83, no. 1-2, pp. 351-358, 2002.

[10] Food and A. O. of the United Nations. (2011) Global observation of forest and land cover dynamics. Internet draft. [Online]. Available: http://www.fao.org/gtos/gofc-gold/overview.html

[11] M. E. Assessment, "Forests and woodland systems," in Ecosystems and human well-being, Volume 1: current state and trends : ndings of the Condition and Trends Working Group, R. Hassan, R. Scholes, and N. Ash, Eds., 2005, ch. 21.

[12] C. Vidal, A. Lanz, E. Tomppo, K. Schadauer, T. Gschwanter, L. di Cosmo, and N. Robert, "Establishing forest inventory reference definitions for forest and growing stock: a study towards common reporting," Silva Fennica, vol. 42, no. 2, pp. 247-266, 2008.

[13] INSPIRE. (2011) Infrastructure on spatial information in europe. [Online]. Available: http://inspire.jrc.ec.europa.eu/

[14] S. Bacharach, “Opengis web map service," Open GeoSpatial Consortium Inc., Standards Track ESE-RFC-005v0.02, 2005.

[15] G. Geller and W. Turner, "The model web: a concept for ecological forecasting," in Proc. of Geoscience and Remote Sensing Symposium, 2007. IGARSS 2007, Barcelona, Spain, Jul 2007, p. 24692472.

[16] P. Mayaux, P. Holmgren, F. Achard, H. Eva, H. Stibig, and A. Branthomme, "Tropical forest cover change in the 1990s and options for future monitoring," Philosophical Transactions of the Royal Society B: Biological Sciences (Phil. Trans. B), vol. 360, pp. 373-384, 2005.

[17] F. Achard, H. Eva, E. Lindquist, A. Bouvet, O. Arino, and P. Mayaux, "Estimating tropical deforestation," Carbon Management, vol. 1, no. 2, pp. 271-287, 2010.

[18] A. Camia, P. Barbosa, G. Amatulli, and J. S.-M. Ayanz, "Fire danger rating in the european forest fire information system (effis):current developments," in Proc. of the 5th International Conference on Forest Fire Research, vol. 1, Figueira da Foz, Portugal, Nov 2006.

[19] A. Camia and G. Amatulli, "Weather factors and fire danger in the mediterranean," in Earth Observation of Wildland Fires in Mediterranean Ecosystems, E. Chuvieco, Ed. Berlin, Germany: Springer-Verlag, 2009.

[20] R. Beuchle, H. E. H. Stibig, C. Bodart, A. Brink, P. Mayaux, D. Johansson, F. Achard, and A. Belward, "A satellite data set for tropical forest change assessment," International Journal of Remote Sensing., p. In Press, 2011.

[21] C. Bodart, H. Eva, R. Beuchle, R. Rasi, D. Simonetti, H. Stibig, A. Brink, E. Lindquist, and F. Achard, "Pre-processing 
of a sample of multi-scene and multi-date landsat imagery used to monitor forest cover changes over the tropics," ISPRS Journal of Photogrammetry and Remote Sensing, p. In Press, 2011.

[22] R. R. si, C. Bodar, H. Stibig, R. Beuchle, S. Carboni, D. Simonetti, and F. Achard, "An automated approach for segmenting and classifying a large sample of multi-date landsat-type imagery for pan-tropical forest monitoring," Remote Sensing or Environment, p. In Press, 2011.

[23] D. Simonetti, R. Beuchle, and H. Eva, "User manual for the jrc land cover/use change validation tool," Publications Office of the European Union, EUR - Scientific and Technical Research Series ISSN 1018-5593, 2011.

[24] J. Cepicky, "Pywps: Grass goes web," in Proc. of Free and Open-Source Software for GeoInformatics (FOSS4G), vol. 1, Lausanne, Switzerland, Sept 2006.

[25] J. Cepicky and L. Becchi, "Geospatial processing via internet on remote servers - pywps," OSGEO Journal, vol. 1, 2007.

[26] M. Neteler and H. Mitasova, Open Source GIS: A GRASS-GIS Approach. New York: Springer-Verlag, 2008.

[27] R Development Core Team, R: A Language and Environment for Statistical Computing, R Foundation for Statistical Computing, Vienna, Austria, 2010, ISBN 3-900051-07-0. [Online]. Available: http://www.R-project.org

[28] L. Diaz and S. Schade, "Geoss service factory: Assisted publication of geospatial content," in Proc. of the 14th AGILE Conference Advancing Geoinformation Science for a Changing World, vol. 1, Lausanne, Switzerland, 2011, pp. 423-442.

[29] M. Nunez Redó, L. D. Sánchex, J. G. Altaba, D. Gonzálex, and J. H. Guijarro, "Discovery and = integration of web 2.0 content into geospatial information infrastructures: A use case in wild fire monitoring," in Proc. of First International Conference on Massive Information Sharing and Integration, Vienna, Austria, Aug 2011, pp. 50-68.

[30] EFFIS, "Forest fires in europe 2010," European Commission Joint Research Centre, Tech. Rep. EUR 24910 EN, 2010.

[31] A. Pekkarinen, L. Reithmaier, and P. Strobl, "Pan-european forest/non-forest mapping with landsat etm+ and corine landcover 2000 data," ISPRS Journal of Photogrammetry and Remote Sensing, vol. 64, pp. 171-183, 2009.

[32] P. Kempeneers, F. Sedano, L. Seebach, P. Strobl, and J. S.-M. Ayanz, "Data fusion of different spatial resolution remote sensing images applied to forest type mapping," IEEE Transactions on Geoscience and Remote Sensing, vol. 1, p. In Press, 2011.

Daniel McInerney is a Research Fellow at the Insitute of Environment and Sustainability of the European Commission's Joint Research Centre. He holds an M.Sc. in Geo-Information Science from the University

PLACE PHOTO HERE of Edinburgh and a Ph.D. from Univeristy College Dublin in the use of optical remote sensing to monitor forests. Prior to joining the EC-JRC, Daniel was a researcher at Urban Institute Ireland \& University College Dublin and at Forest Research, Edinburgh, Scotland. His research interests include geo-information systems, web processing services, remote sensing applied to forest monitoring. 
Lucy Bastin is currently a Visiting Senior Scientist at the JRC from 2010 - 2011 working on the generation of web-based validation tools for use by an international community of experts in biodiversity and forestry

PLACE

PHOTO

HERE monitoring. She holds a Senior Lectureship in the School of Engineering and Applied Science at Aston University, UK, where she applies spatio-temporal analysis techniques to challenges in conservation planning, infection monitoring, and other environmental and socio-demographic contexts. She is also an investigator on the FP7-funded UncertWeb project, which addresses the management, reliable use and transfer of uncertainty information within a distributed, interoperable ModelWeb.

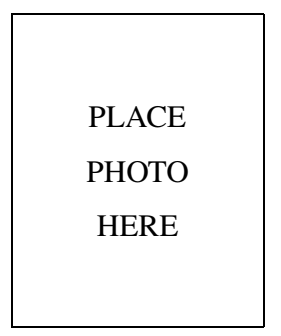

Laura Díaz graduated in Computer Engineering (2000) from the Universitat de València and obtained her MSc in Intelligent Systems (2008) and the doctoral degree in Geospatial Science (2010) from Universitat Jaume I of Castelln where she is currently a research associate. She has participated in R\&D projects at the Institute of Robotics at the Universitat de València and GIS companies such as Geodan (The Netherlands) and Iver TI (Spain). Furthermore, she participated in research stages at Institute of GeoInformatics (University of Münster) and Institute of Environment and Sustainability (Joint Research Centre).Her main research interests are in the field of Geospatial Information Infrastructures and User generated content, in particular she investigates novel approaches for improving geospatial content integration and publication, distributed geoprocessing and service interoperability.

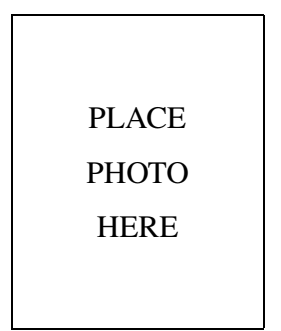

Jesús San-Miguel Ayanz received the B.S. degree in forest engineering from the Polytechnic University of Madrid, Madrid, Spain, in 1987 and the M.Sc. and Ph.D. degree on remote sensing and geographic information systems from the University of California, Berkeley, in 1989 and 1993, respectively.

He was with the University of Cordoba, Spain, where he was an Assistant Professor from 1994 to 1995 and an Associate Professor of forest inventory, forest mensuration, and remote sensing from 1995 to 1997. He was also a Research Fellow with the European Space Agency, Noordwijk, The Netherlands, from 1993 to 1994, and with the University of California from 1989 to 1993. He is a Senior Researcher with the Institute for Environment and Sustainability, Joint Research Centre, European Commission, Ispra, Italy, in the field of forestry. He is also Leader of the FOREST Team. 


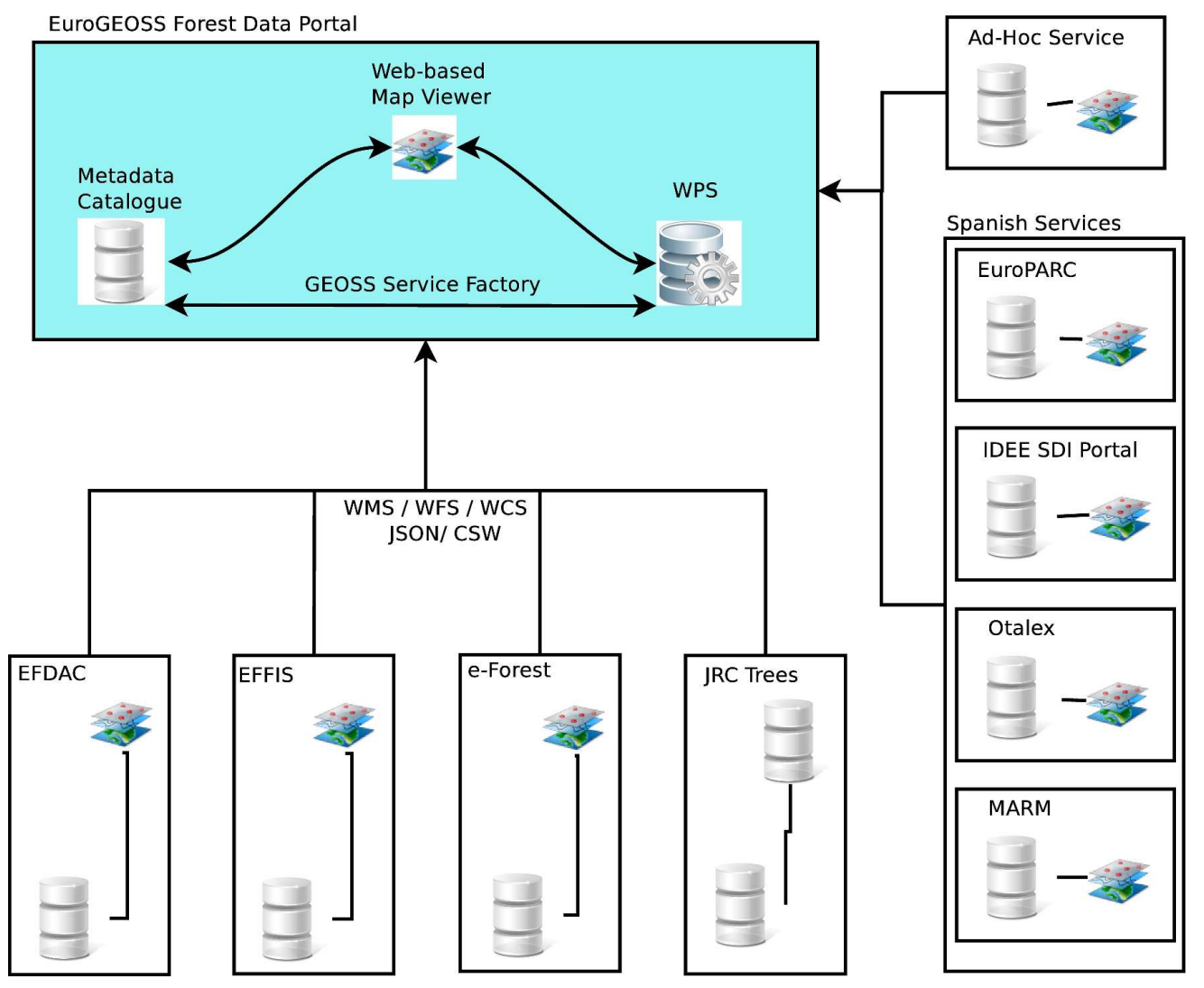

Figure 1. Architectural Overview of Forest Web Viewer and Metadata $300 \times 246 \mathrm{~mm}(300 \times 300 \mathrm{DPI})$ 


\section{Page 19 of 22}

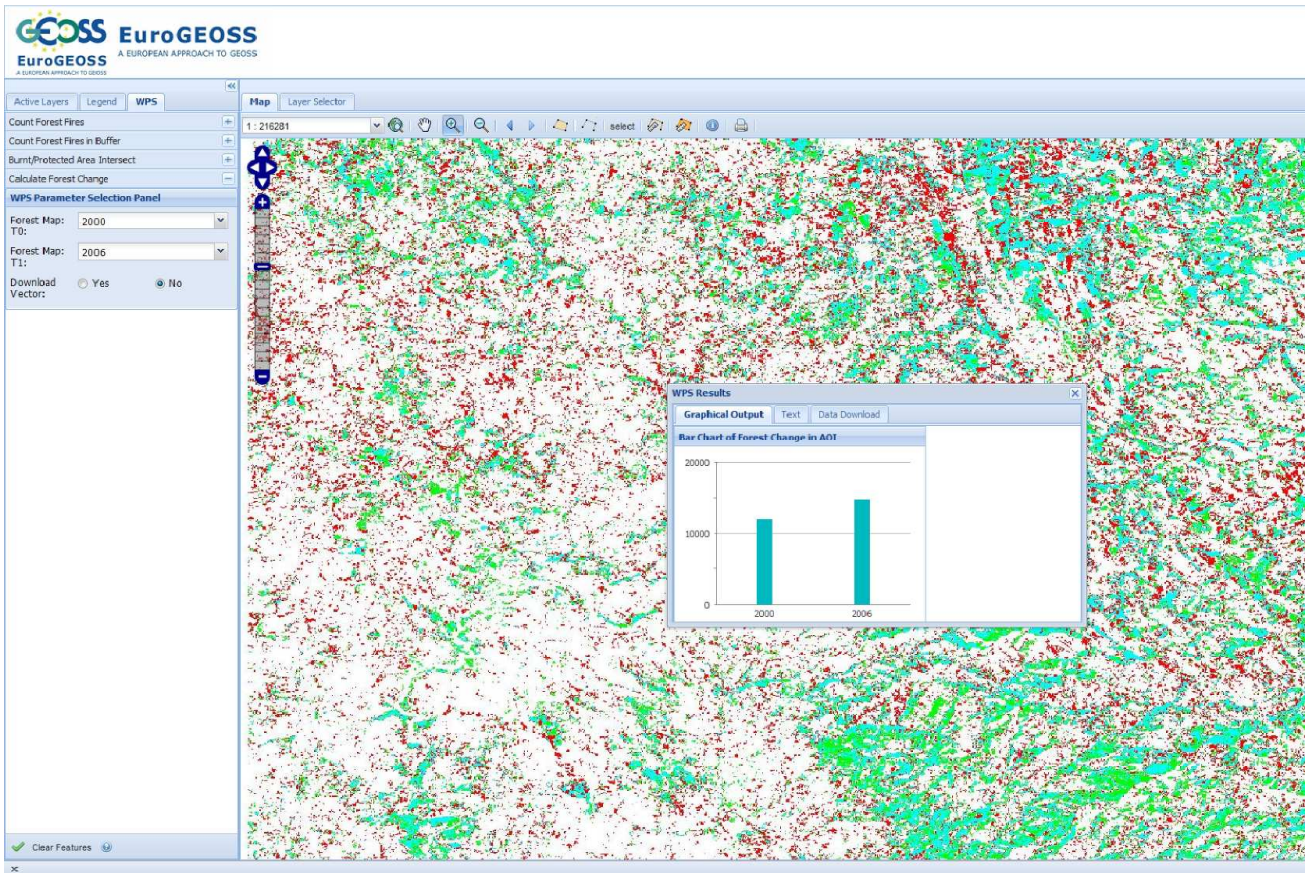

EuroGEOSS Forest Web Viewer with WPS outputs $282 \times 188 \mathrm{~mm}(300 \times 300 \mathrm{DPI})$ 


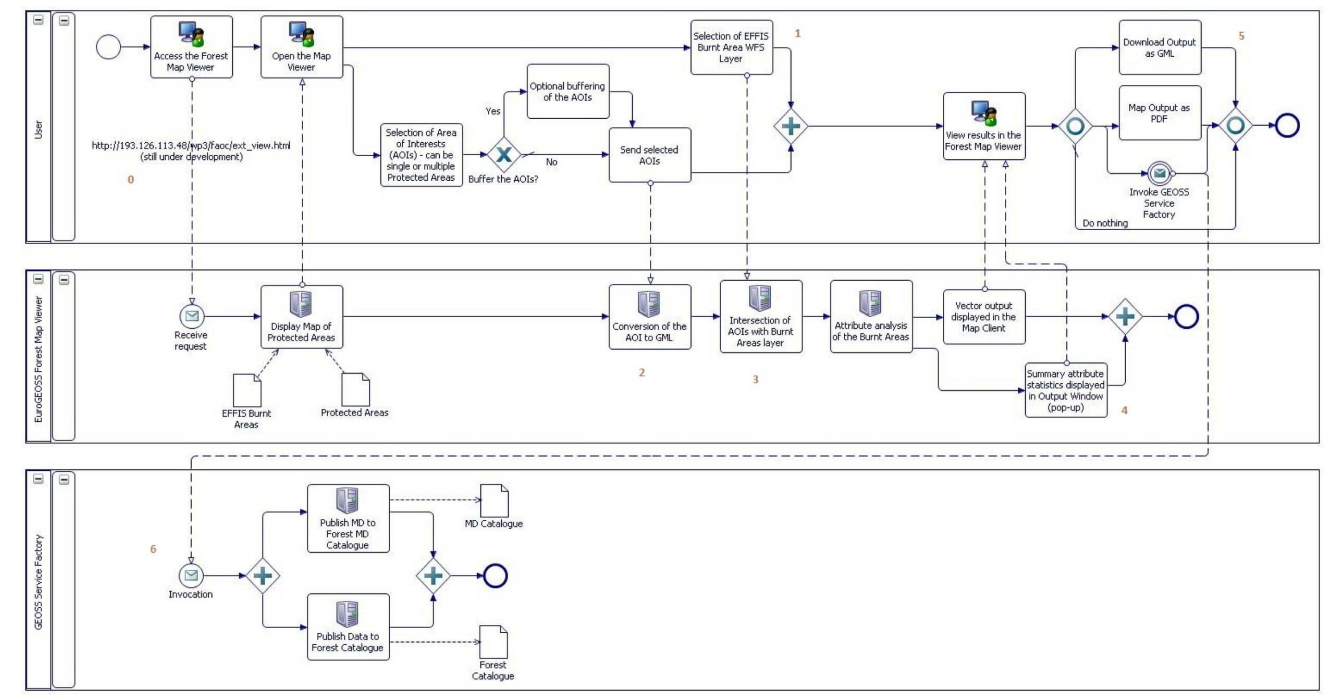

Figure 3 BPMN Diagram of burnt area analysis $211 \times 112 \mathrm{~mm}(300 \times 300 \mathrm{DPI})$ 
Page 21 of 22

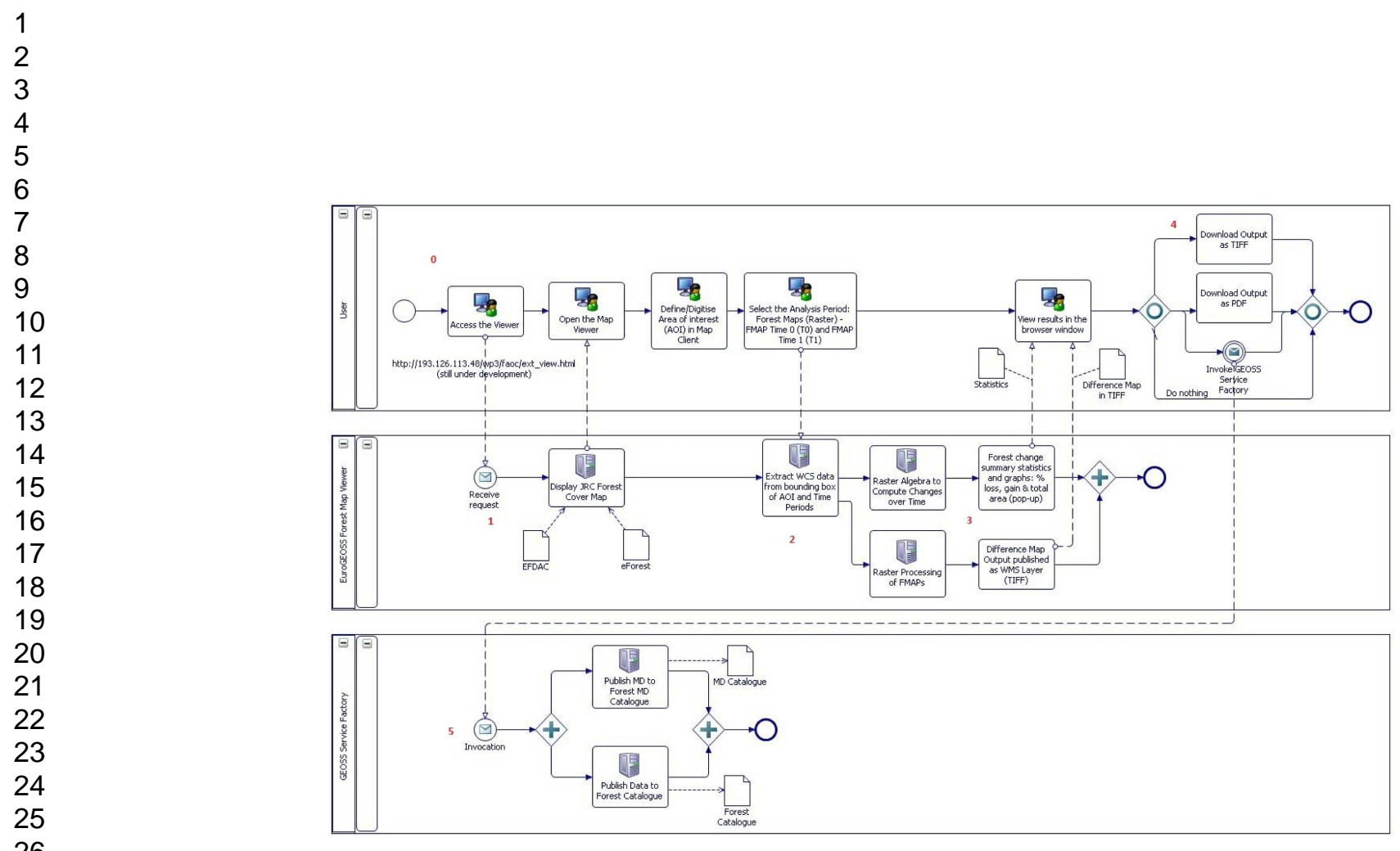

Figure 4 BPMN Diagram of forest change analysis $212 \times 127 \mathrm{~mm}(300 \times 300 \mathrm{DPI})$ 


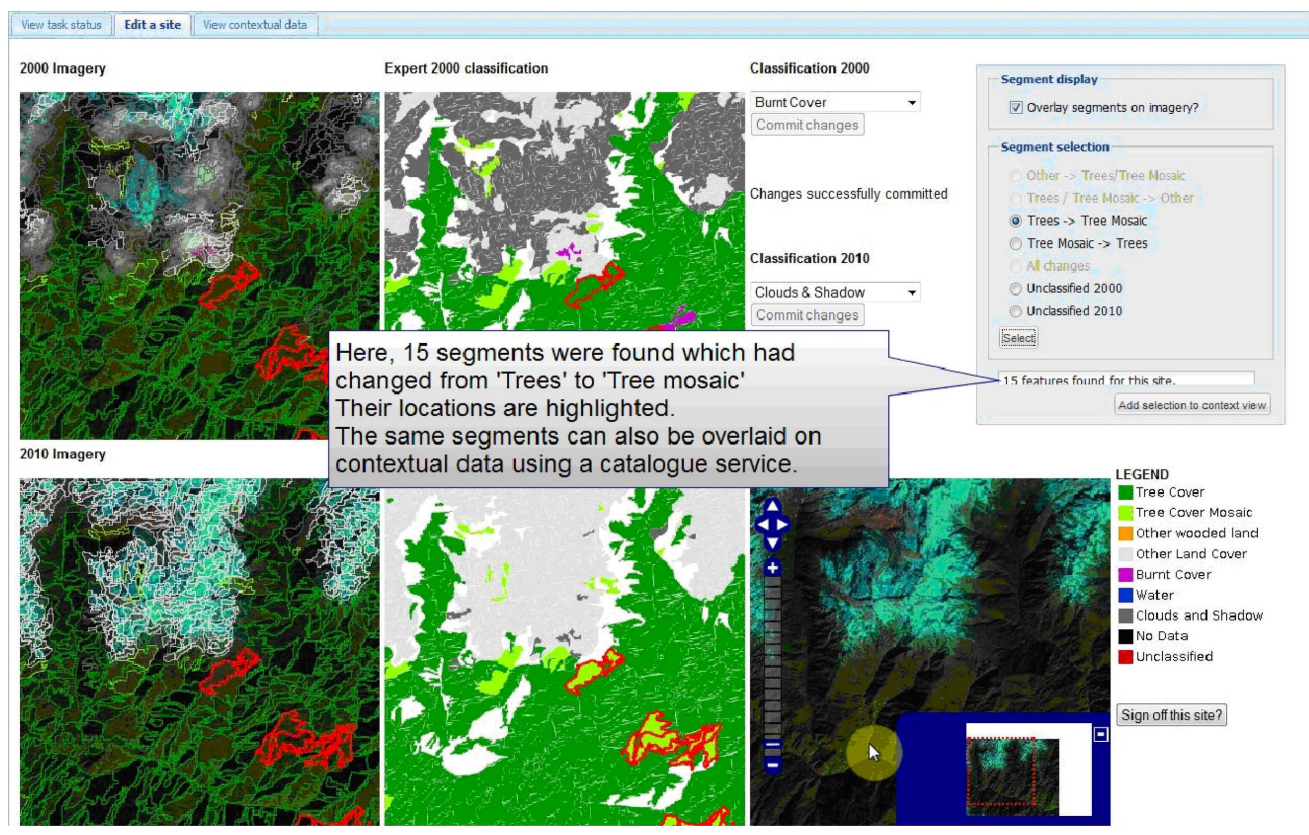

Figure 5 Landcover validation Editor $209 \times 131 \mathrm{~mm}$ (300 x 300 DPI) 